\title{
4 Social uses of the Internet and the construction of new models for mathematics teaching and learning
}

\author{
Carles Romero i Chesa \\ IES Manuel Blancafort \\ La Garriga \\ Barcelona, Spain
}

Ferran Ruiz i Tarragó

Generalitat de Catalunya

Departament d'Ensenyament, PIE

Barcelona, Spain

\begin{abstract}
Educational implications of information and communication technologies are far more reaching than initially expected. Present models of mathematics teaching are quickly becoming obsolete because the new paradigms brought by the Internet enable new modes of mathematical thinking and new contexts of meaning. The structure of mathematical discourse is affected by visualisation and distance interaction, so there is a strong need to reorganise didactic sequences and to define new curriculum objectives taking into account the variety of modes of mathematical thinking that were not feasible in the past but which can now be sustained by the technology.
\end{abstract}

\section{Keywords}

Cognition, constructivism, curriculum development, Internet, social issues. 


\section{INTRODUCTION}

Information and communication technologies epitomise in many aspects the world of the last quarter of the $20^{\text {th }}$ century and have become one of the building blocks of modern societies. On the whole, educational systems are making huge efforts to cope with these technologies, integrating them in order to develop students' capabilities and new teaching methods, or, in some cases, merely so as not to be left behind in terms of technical and economic progress.

These technologies, like any other, are initially developed with specific goals in mind, to solve a problem or a family of problems, and are applied by people and organisations in the pursuit of economic advantages, personal convenience or mastery of nature. Owing to changes in the environment, however, they produce effects beyond those initially anticipated. Technological changes often give rise to unattended or previously unimaginable results that can be much more farreaching than those originally expected or desired. There already seems to be reasonable evidence that this also applies to the integration of information and communication technologies into education (Ruiz, 1993), and that in mathematics it acquires very specific characteristics that can lead to new models of teaching and learning and even to a renewal of curriculum objectives.

To summarise, telecommunications are giving rise very quickly to new economic and social relationships, and evidence gathered so far would seem to suggest that new teaching and learning paradigms are also emerging. So it is necessary to avoid a naiive approximation to the technological change when trying to ascertain the effects and implications of computers and the Internet on mathematics education.

\section{SOCIAL USE OF THE INTERNET AND MATHEMATICS}

The use of the plethora of telecommunication networks known as the Internet show some common patterns. Apart from commercial and business applications, Internet is mainly used to support and facilitate interpersonal communication; to search for information and to retrieve it in a variety of forms, although text is still the predominant mode; to carry collective or public discussions that can lead to collaborative construction of knowledge; and to publish multimedia materials, making them available to the whole Internet community. These four paradigms of the social use of the Internet can foster curriculum development, benefit teaching activities, and improve student's learning in mathematics as well as in other curriculum subjects, although its implementation and use in secondary education is still in its infancy. 


\section{Interpersonal communication}

People all over the world use the Internet's electronic mail facilities to exchange information and to establish meaningful dialogues with other people on a personal basis. Teachers and students can take advantage of the e-mail as it is cheap and easy to operate: pupils can send completed assignments in electronic form to their teachers; the assignments can be marked and returned to students very quickly and with personalised comments; students in different locations can participate in collaborative projects; and distance barriers cease to be a problem.

\section{Information provision and retrieval}

There is a whole world of information in the Net, and there is frequent use of the browsing and retrieval facilities of the World Wide Web (WWW). At professional levels, 'electronic journals' that still maintain a quite traditional layout and design are a cheaper and fastest alternative to mathematical journals. As Okerson (1996) points out, this type of use is probably going to grow and its scope to be more widespread, setting new social problems.

It is already possible to search on-line mathematical dictionaries, to access facsimiles of original papers of great mathematicians, to obtain information on the history of distinguished mathematical objects and to locate the biographies of great authors. There is a growing richness of Web pages with collections of problems at almost any level, and the implementation of Java and VRML languages allows the 'netsurfer' to access galleries of dynamically manipulable mathematical objects'.

\section{Public discussions}

Public discussions consist of specific virtual that are freely joined by people with common and specific interests. In the mathematics field there are 'classical newsgroups' like sci.math, sci.math.*, and alt.algebra.help and 'mailing lists' such as owner-mathqa@lists.oulu.fi. The Internet holds the record public discussions on mathematical issues maintained by groups of students of higher levels - recently joined by senior or retired mathematicians - that enjoy answering questions of younger students and stimulating them with new challenges. An example here is the virtual $D r$. Math who treats mathematical illnesses'.

Public discussions allow for collaborative knowledge construction in many areas, and 'Frequently Asked Questions' (FAQ) boards are often built up by means of the collaborative efforts of a large number of people.

\section{Publishing on the Internet}

The Internet provides a means for publicising the results of many creative acts of people that could not reach public audience by means of traditional publishing channels. This applies to many areas of human creativity, and specifically to mathematics and mathematical education. To publish on the Internet is becoming easier and easier, and almost anyone can post their papers, essays, personal 


\section{Information and communications technologies in school mathematics}

databases and multimedia products. The personal touch or style of the author in presenting information is not hindered by rigid publishers' criteria, it is only shaped by common operating procedures.

\section{The Internet and mathematics}

Both in concepts and in technologies, the Internet is a rapidly evolving reality. Quoting the UK's National Council for Educational Technology (NCET, 1995), present communications technologies already

"provide students with opportunities for using and applying mathematics in the solution of problems, helping to develop mathematical reasoning, and enriching work with number, shape, space and measures. In the area of handling data, in which students establish lines of enquiry and collect, analyse and interpret data, the Net can be particularly useful". (Page 13)

It is nevertheless necessary to recall that in the early stages people tend to use computers to reproduce tasks which were done before without them. They are used to get better presentations, to perform faster calculations, and to provide enhanced examples for the usual curriculum subjects. As Bottino and Furinghetti (1994) point out, this approach is deeply rooted in the didactic paradigms teachers have so far, paradigms that are currently being undermined by the technology. There seems to be a similar trend in the fast-expanding process of using computer-mediated communications in education,

It is necessary to keep in mind that any new technology causes the rules of society, institutions, people's roles, and their relationship with the environment to be rebuilt to some extent (Schwarz, 1992). Generalised computing and communication facilities will have many impacts on mathematical education. Therefore, research and debate on the new tendencies and paradigms of mathematics teaching and learning is needed.

On the other hand, the use of the Internet is spreading very quickly in many fields of human activity, specifically in those of research, business and production. A huge social demand for people with information processing and communication skills is correspondingly emerging. This is by no means a small issue in mathematical education as we will attempt to illustrate.

\section{MATHEMATICS: WHAT ARE WE TALKING ABOUT?}

Paraphrasing Wittgenstein we ground our arguments in the idea that 'mathematics' is not a doctrine but an activity. So it is necessary to establish which activities can be considered as 'mathematical activities'. Critical analysis of the history of science demonstrates that the meaning of the term mathematics has changed diachronically, and it is synchronically variable within the context. Thus, for instance, the actual classification of the Mathematical Review is useless 
to classify the items of mathematics curricula of primary and secondary education. So our approximation will take into account two points of view: modes of mathematical thinking and contexts of meaning.

\section{Modes of mathematical thinking}

First, it is necessary to ascertain whether a given mode of thinking can arguably be called 'mathematical', with criteria wide enough to include not only academic mathematics but the mathematics of all levels of human activity.

Tall's classification (Tall, 1994) is helpful here in that he has classified the modes of mental representation of mathematical objects and activities upon these objects. The most primitive is the enactive sensori-motor mode, which is at the root of much of our activity. It begins with perceiving objects in the external world and acting upon them; objects can be handled and demonstrations made by means of direct physical experimentation.

The iconic visuo-spatial mode uses iconic representations of objects, enabling us to sense spatial relationships to elaborate and interpret pictures and to consider thought experiments (what if ...?) as a demonstration method, for example, we may imagine holding certain conditions and 'see' whether a conclusion should follow.

In the (visuo-)symbolic mode, one can distinguish:

a) the symbolic-verbal mode of the classical Euclidean geometry in which, while conforming to the concept of Euclidean demonstration, objects are described and idealised and relationships are verbalised;

b) the proceptual mode (procept-processtconcept) of the algebra and elementary calculus; and

c) the symbolic-logical mode, in which objects are defined, relationships are inferred and demonstrations are formal.

As an illustration, the New Maths didactic paradigm was rooted on the symbolic-logical mode of thinking. Linear concept formation is intrinsic to it and, correspondingly, student's first perceptions of mathematical objects are microscopic.

Information technology fosters a vigorous trend to consider visualisation as a legitimate way of approaching mathematical objects with students' perceptions becoming that of macroscopic instead of microscopic. Moreover, the capabilities of the computer introduce a new element, the manipulation of a representation. This suggests the inclusion of a new enactive mode of thinking, similar to the one described above but with a new characteristic: instead of objects being real only in terms of physical entities, to be handled physically, they become virtually real in that the representation and manipulation become more dynamic and flexible in the computing environment. 


\section{Mathematics and contexts of meaning}

It can be said that a mathematical activity belongs to 'pure' mathematics when the objects dealt with and the processes done with these objects have meaning exclusively on mathematical contexts. But the social importance of mathematics arises from peoples' capabilities in applying modes of mathematical thinking to very different fields of human experience and activity. It may seem obvious to point out that our societies are in many respects sustained by the mathematical capabilities of its people. Although people have always developed the skills needed to manipulate emerging technologies, nowadays we are facing a new challenge: informatics and telecommunication technologies are immensely more complicated and the models necessary to operate them have become more and more abstract.

Computers and global networks are related to mathematics not only by their capabilities of quick and accurate computing, and the need to operate them according to logical rules, but because people efficiently use these technologies without needing to know about their internal working, nor their structure and the concepts underlying the development of the software (Keitel et al., 1992). On the other hand, the ability to interpret and ascribe meaning to the human operation of computers and networks implies the need for elaborate abstract mental models and a grasp of the internal logic which underpins the interactions. These are genuine mathematical skills to be fostered by appropriate teaching.

The implications are that in our society, people responsible at every level of mathematical education should reflect on and research the possibility of opening up the closed arena of mathematical education to include areas and contexts other than the traditional.

The field and objectives of mathematical education should accordingly be extended to new areas, and specifically to the techniques of processing and transmitting information: it doesn't seem exaggerated nor visionary to insist on the stable introduction of such subjects as modern programming techniques, database design and query languages, and graphical representation techniques in secondary education.

\section{THE CONSTRUCTION OF NEW MODELS FOR MATHEMATICS TEACHING AND LEARNING}

\section{On the structure of mathematical discourse}

Pre-Hellenic mathematics is usually considered as a set of cumulative, semiempirical and disconnected practices validated by centuries of daily practice. The Greeks are credited as the inventors of the way we make mathematics, with Euclid's Elements as the paradigm. From then on the mathematical discourse has been linear and implemented as a text. Hilbert's formalism is the highest expression of this approach: it gave rise to the 'New Maths' didactic paradigm of 
the fifties and sixties. A very special characteristic is the absence of illustrations; the linear deductive logic dominates the discourse so that mathematics becomes a form of text. Pictures and diagrams are absent because they can be read as nonlinear networks of relationships. Even today, many teachers expect their students to elaborate linear discourses reflecting the Bourbaki style.

However, a new tendency is emerging: the mathematical ability of students is proportional to the quantity of meaning of their conceptions. Visualisation is a source of meaning in the perception of mathematical objects (Zimmerman and Cunningham, 1991). The capabilities of computers and networks in this area are continuously growing, and it is already possible to construct visual representation of objects that were never before represented in such a way, and at the same time providing a means for illustrating the network of their internal relationships. On the Web, for example, classical geometry is illustrated and manipulated by means of a dynamic implementation of Euclid's Elements using Java applets ${ }^{3}$. The impact of that fact is probably going to be much greater and global than, for instance, Argand's representation of complex numbers. This fact, along with the generalisation of the hypertext, will probably make the exigency of a linear mathematical discourse obsolete and intolerable.

\section{The computer as a tool to enhance meaning}

Computers were initially employed to facilitate computing and representation tasks previously considered too long or too difficult, but it seems that these uses have had little impact on mathematical education, although a few research areas did open up because of insights prompted by the computer.

Proposals to place algorithms in a central position in school mathematics are at least twenty years old. The proceedings (Johnson and Tinsley, 1978) of the 1977 IFIP Working Conference on informatics and mathematics contain many authors arguing on mathematical concept formation and problem solving capabilities that benefit from algorithms' analysis, construction and implementation. Nowadays there is considerable empirical evidence of the successful use of the computer to enhance meaning using programming languages specifically designed to convey mathematical processes, or by building up environments to construct and explore mathematical concepts (Dubinski and Tall, 1991).

But, in the near future, students' perceptions of mathematical objects are going to be quite different from what teachers still expect today, because they will take place in very different environments. Consequently, educational use of computers and networks is leading to a new perception of mathematical objects.

From a general point of view, current literature accepts widely that emerging didactic paradigms are rooted on the developments of Vygotski and Piaget, which stress the importance of social interaction and of interaction with the environment. Consequently, networked computers provide a framework of huge power, which, when fostered by appropriate teaching, can empower learner's building up of mathematical knowledge. 
Reorganising didactic sequences and the curricula

Information and communication technologies open up many new possibilities. It is possible to change substantially the methodological approaches of the 'pure mathematics' field. Without the computer it is necessary to routinise algorithms and methods first, and only then does it become possible to manipulate a mathematical object with enough sophistication to be able to cope with its whole meaning. But now, as Tall (1994) points out, it is possible to reverse this process, and make the student use specific software to first conceptualise the object and understand its properties, and later, develop the manipulation processes that are intrinsic to that object. Thus, for instance, in order to learn the concept of the derivative of a function it is possible to use a special purpose piece of software such as Graphic Calculus (Tall et al., 1990) or use a more general purpose program for the graphical representation, e.g., Derive. Successive magnifications of the behaviour of the graphic around one point demonstrates dynamically the fact that the curve is approaching a straight line that doesn't change in further close-ups. The concept of derivative of a real function arises then in a very natural form, but there is more than this. One can do the same process with non differentiable functions at a fixed point or at any point, and students will see that they are never able to obtain a straight line. The concept image of the student is now provided with examples and counter-examples of the concept of differentiability in a way that cannot be done without the machine, and all before any formal definition of the concept of derivative and the acquisition of techniques for differentiation.

Telecommunications add new dimensions and capabilities for enhancing meaning brought about by powerful visualisation techniques. Experimental Web sites implemented in languages such as VRML demonstrate these capabilities in the study of polyhedra ${ }^{4}$ or the space geometry, examples of ill-fated areas in the traditional curricula. Furthermore, it is not necessary to run a finished and properly marketed program installed in one's own PC. The Internet allows developers to create prototypes and applications that don't need to be restricted to a single computer, i.e., sets of applets placed elsewhere in the network can be linked together, thus enabling multiple forms of interaction.

\section{CONCLUSION: TOWARDS A NEW DEFINITION OF CURRICULUM OBJECTIVES}

It can be rightly argued that, although the new environments enable the building up of very meaningful concepts, they cannot represent the general concept. When students manipulate geometric constructions with a Java applet or with a tool like Cabri Géomètre they can obtain a deep understanding of the properties of the objects and their relationships. But the thinking mode of Euclid's geometry 
requires one to be able to describe the objects and to verbalise their properties in order to realise the Euclidean demonstration of proof. In a similar way, the enactive action over functions and slopes doesn't necessarily lead to the logicsymbolic mode which characterises academic mathematics, in which objects are defined, relationships are inferred and demonstrations or proofs are formal.

Is it necessary then to infer that computers are only helpful in very specific or elementary situations of mathematical education? Taking into account that 'mathematical education' is a broad concept that refers to many issues, we postulate that when defining a priori the objectives of a specific curriculum, it is necessary to bring to the foreground the issue of the selection of modes of mathematical thinking, in order to decide which modes should be fostered or which ones should be ignored. Two main reasons are put forward here in support of this position.

Firstly, information and communications technology constitutes in itself a deep change of the didactic environment, and a very important element in this change is its capacity to foster new forms of authentic mathematical thinking that cannot be accomplished in the traditional environments. Now it is possible to engage in activities which were not even considered in the past, when 'doing' mathematics was limited to what could be done with paper and pencil. It is proposed here that this will provide 'a breath of fresh air' for those students who now feel uncomfortable with the classical paradigm of mathematical learning which, with its current heavy emphasis on deduction, may seem to be lacking in its attention to exploration and experimentation.

Secondly, although our societies rely on the mathematical skills of their people, it is useless to expect that a sizeable part of the population will accept and share in a mathematical culture whose final criterion is the internal consistency agreed between mathematicians (Skemp, 1971). Correspondingly, curriculum goals now need to be defined within a wider perspective because technology, for instance, allows the learner to approach geometry without the requirement of a formal demonstration language, or to work with the calculus without mastering derivation and integration rules. Legitimate and different mathematical interests can now be linked with different modes of mathematical thinking. Consistency can be a matter for agreement between teacher and learner engaged in a meaningful dialogue mediated by the working environment, that is, by the new techniques of information processing and the emerging paradigms of communication brought about by computers and the Internet. 
38 Information and communications technologies in school mathematics

\section{REFERENCES}

Bottino, R. and Furinghetti, F. (1994) Teaching Mathematics and Using Computers: links between teachers' beliefs in two different domains. In J. da Ponte and J. Matos (eds.) Proceedings of the Eighteenth International Conference for The Psychology of Mathematics Education, II, 112-119. Lisboa.

Dubinsky, E. and Tall, D. (1991) Advanced Mathematical Thinking and the Computer. In Advanced Mathematical Thinking, Kluwer Academic Publishers, Dordrecht, 231-248.

Johnson, D. and Tinsley, D. (eds.) (1978) Informatics and mathematics in secondary schools: Impacts and relationships, North-Holland.

Keitel, C., Kotzmann, E. and Skovsmose, O. (eds.) (1992) Beyond the Tunnelvision (Mathematics-Society-Technology). In Learning from the Computer, $1-48$.

NCET (1995) Highways for Learning - An introduction to the Internet for schools and colleges. ISBN 1853793183 . Also available on the Internet ${ }^{5}$.

Okerson, A. (1996) Whose article is it anyway? Notices of the American Mathematical Society, 43(1), 8-12

Ruiz, F. (1993) Integration of Information Technology into Secondary Education - Main Issues and Perspectives. IFIP Working Group on Secondary Education WG 3.1, IFIP Secretariat, Geneva.

Schwarz, M. (1992) Technology and Society: Dilemmas of the Technological Culture. Proceedings of the Third European Congress on Technology Assessment 'Technology and Democracy', 1, 53. Copenhagen.

Skemp, R.R. (1971) The Psychology of Learning Mathematics. Penguin Books.

Tall, D., van Blockland, P. and Kok, D (1990) A Graphical Approach to the Calculus.. Rivendell Software, 21 Laburnum Avenue, Kenilworth, Warwickshire CV8 2DR, UK.

Tall, D. (1994) A Versatile Theory of Visualisation and Symbolisation in Mathematics. Invited plenary lecture at the CIEAEM Conference, Toulouse, France, July 1994.

Zimmermann, W., and Cunningham, S. (eds.) (1991) Visualization in Teaching and Learning Mathematics. MAA Notes, 10, 224. The Mathematical Association of America, Washington.

\section{INTERNET WEB SITE REFERENCES:}

1. http://www-groups.dcs.st-and.ac.uk/ history/BiogIndex.html http://www.scottlan.edu/lriddle/women/women.html http://www-groups.dcs.st-and.ac.uk/ history/Java/index.html http://www.li.net/ george/virtual-polyhedra/vp.html 
2. http://forum.swarthmore.edu/dr.math/dr-math.html

3. http://aleph0.clarku.edu/ djoyce/java/elements/elements.html

4. http://www.li.net/ george/virtual-polyhedra/vp.html

5. http://www.ncet.org.uk/publications/highways/

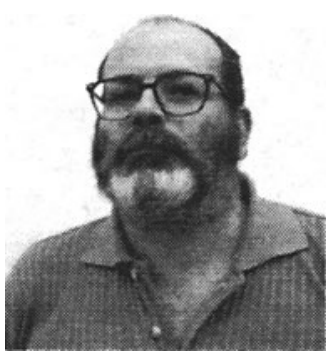

Carles Romero i Chesa is a physics graduate and head of the mathematics department of the I.E.S. Manuel Blancafort of La Garriga, Barcelona. He is Magister in Research on the Didactics of Mathematics by the Universitat Autònoma de Barcelona (1993) and member of the project Advanced Mathematics Thinking Processes of this university. He coordinates the Web pages on mathematics of the Programa d'Informàtica Educativa and is author of several papers on mathematics education. Other interests are mathematics social perception, Java programming and playing organ music.

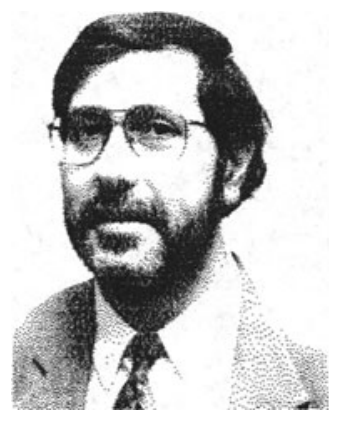

Ferran Ruiz i Tarragó holds a physical sciences degree from the University of Barcelona, with postgraduate studies in Methodologies and Management of Training of Trainers. He has been director of the Programa d'Informàtica Educativa, Information Technology in Education Programme of the Department of Education of the Generalitat de Catalunya (Catalunya's Autonomous Government) since 1994; member of Working Group 3.1 of the International Federation for Information Processing (IFIP) since 1990. He won the EPSON Award on information technology in education in 1990 and is author of more than twenty papers and publications on information technology in education, including IFIP WG 3.1: Guideliness of Good Practice: Integration of Information Technology into Education: Main Issues and Perspectives. 